\title{
THE POLISH IMPLEMENTATION OF DECOMPRESSION PROCEDURES IN UNDERWATER DEEP-SEA WORKS PART I. REVOLUTION OF PERSONAL DIVING COMPUTERS
}

Stanisław Skrzyński

Faculty of Mechanical and Electrical Faculty, Polish Naval Academy in Gdynia, Poland

\section{ABSTRACT}

In the article the author presents the specificity of deep diving decompression in relation to the revolution in diving which was triggered by the introduction of a personal computer in recreational diving. Continuous development of these computers, especially in the last decades of our century, concerned deep-sea technical and recreational dives. Deep-sea dives are difficult and risky in terms of underwater physiology and decompression. In characterizing the decompression of a diver, realized with the use of a computer for deep-sea dives, the author compares the realization of decompression in professional deepsea dives. He also points out the formal and technical obstacles to the implementation of personal dive computers. In summary, the article evaluates the possibility of using the diving computer in the implementation of underwater works.

Keywords: diving computer, application of diving computer diver's decompression, deep underwater work, deep diving, professional diving, data deep diving, diving bell, breathing mixtures, decompression table.

\section{ARTICLE INFO}

PolHypRes 2019 Vol. 68 Issue 3 pp. $41-58$

ISSN: $1734-7009$ elSSN: $2084-0535$

Rewiev article

DOI: $10.2478 / \mathrm{phr}-2019-0012$

Pages: 18 , figures: 0 , tables: 0

page www of the periodical: www.phr.net.pl

Publisher

Polish Hyperbaric Medicine and Technology Society
Submission date: 27.01.2019 r.

Acceptance for print: 13.09.2019 r. 


\section{INTRODUCTION}

Decompression is the last phase of diving, which is decisive for the safety of the diver. Decompression in dives is performed on the basis of decompression tables interpretation, which does not cover all aspects and cases encountered in practice. The decompression process, due to the phenomena occurring during its course in the tissues of a living organism, requires reliable and precise methods of measuring decompression parameters. Therefore, during diving we aim to reproduce decompression tables in the most scrupulous way, taking into account the permissible inaccuracies in parameters and implementation errors.

The revolution in diving computers has not yet taken hold in professional dives on such a scale as it has in technical and recreational dives, although the advantages of using a personal dive computer are clearly evident in both of these forms of diving [1]. Formal documents and procedures still do not recommend the use of a dive computer for professional deep dives. Currently, when planning deep dives, the underwater works manager relies on "paper" decompression tables, the technical possibilities of their implementation and his experience. Operation without computer support is required, following only the "good diving practice".

A practicing underwater manager usually has his or her "secret" habits, visible in his or her approach to decompression, especially during deep-sea diving, which often necessitates implementation of a multi-variant and multifaceted decompression process. Professional and scientific literature very scantly describes these aspects, if at all does it address the problems of decompression, especially in complex diving systems with the use of a bell. The same method of decompression can be realized in many technical and organizational variants, necessitated by the technique, divers' qualifications or the characteristics of the existing diving base. On the basis of the author's contacts with numerous specialistspractitioners in the field of decompression, he came to the conclusion that the knowledge of its implementation is one-sided and does not take into account the entire kaleidoscope of issues that may occur during its course.

When asked how decompression should be carried out correctly, the authors of decompression tables, their interpreters and diving specialists reply by stating that it is obvious that "it should be carried out according to tables", which is difficult to see as a comprehensive answer. They sometimes supplement it by saying that "take care of the diver's safety and ensure that it is not reduced or endangered at any time". In these discussions, the notion of diver safety concerns the direct effects during diving and the so-called distant effects, resulting from the practice of the profession of deep-sea diver, are omitted. In both cases, safety and quality of decompression are affected. In such situations, it is not easy to engage in a deeper discussion, as the arguments of the opponent are based on the interpretation of regulations, norms and personal contacts, and less frequently on their own practice.

No one communicates knowledge about their own mistakes and shortcomings, but instead tries to highlight their own achievements and successes. These facts persuaded the author to share his own experience and conclusions, which he came to through difficult relationships with the diving community. The author actively participated in numerous dives over the years, in all diving positions in the diving team. According to the gradation of positions in the reverse gradation of importance and responsibility: the organizer of underwater work, a researcher working on and implementing the problems of diving technology, technology and organization of deep-sea diving in Poland, the manager of underwater works, and deep-sea diving. He was also a participant in the introduction of newer and newer diving technologies over the last 50 years, and an active observer of their impact on all aspects of diving.

Wishing to convey his accumulated knowledge and insights into the various aspects and problems he encountered during these years, which are often overlooked in professional literature and diving procedures, the author writes this article in the hope that it will serve to broaden the knowledge of future and present generations of divers and underwater work managers and organizers.

\section{GENERAL INFORMATION} DECOMPRESSION IN DEEP DIVES

Decompression tables impose adequate technical and medical protection and combining them organisational elements. Technical and organizational interpretation of the use of tables should impose the accuracy of their implementation, and obligatorily the accuracy of measurement of the basic parameters such as depth, time and preparation of breathing mixtures. The accuracy of measurement should show what is the safety corridor of decompression implementation. There are many aspects, from the method of validation of tables, measurement method and environmental conditions, to the characteristics of the diver's work. From a realistic point of view, deep-sea dives are the most difficult among all types of dives. The aspect that best illustrates the difficulty of these dives is the limitation of the relatively short duration of a diver's stay at a depth (50-90 minutes) caused by the limitations of decompression, i.e. the relatively long time of its implementation, exchange of breathing mixtures and the need for oxygen decompression in its last phase $[2,3]$.

In Polish professional deep dives safeguarding underwater deep-sea works, along with the development of science and technology and the needs of the national economy and defence, decompression was based on foreign tables, or their domestic adaptations, according to the development and introduction of new techniques. The only exceptions are the current official state tables in force, which necessitated the adaptation of the technology to their requirements.

In the case of decompression, the literature on the subject presents it from the medical and organizational point of view, laying down the requirements for changes in depth in time without indicating precisely the technical interpretation. Despite the fact that decompression tables in the world are developed by teams in which the leading role is played not only by doctors, it has been agreed that in diving, specialists in underwater medicine are in charge of decompression of divers, are responsible for the verification of tables, development of therapeutic procedures, and medical procedures [4]. National formal and legal documents clearly state that the underwater work manager is responsible for the course of diving. The 
national formal document [5] does not precisely define the role of the doctor during deep-sea diving, although his/her presence in the diving team is required. The responsibilities of the physician in the team should be determined by the underwater work manager, specifying the scope of the physician's intervention in the course of diving, especially in emergency situations.

In scuba diving, the parameters we operate with during decompression include:

- depth,

- depth (pressure),

- diver's stay time at depth,

- composition of breathing and decompression mixtures,

- rate of changes in ascent and descent depths,

- time of exchange of breathing mixtures,

- time of their use at a given depth,

- time of breathing with oxygen,

- technical possibilities of the applied technique and respiratory diving equipment.

The values of the above mentioned parameters depend on the working depth and time of a diver's stay at a given depth, selected from the decompression tables. In most tables, the time of a diver's stay at a depth, which determines the choice of decompression, is counted from the moment of starting the dive or half of the time of the dive until the moment of starting the ascent from the working depth, i.e. commencement of decompression $[6,7,3]$.

The approach to deep-sea diving technology in the aspect of "applied technique and organization decompression tables - decompression process" is a multifaceted issue, enabling to obtain many system solutions. Particularly important is the problem of choosing the decompression method for a specific diving system, in connection with its safety and efficiency. An optimal diving system requires specific working conditions for the diver and crew, and the provision of adequate, reliable technical facilities to protect the controlled course of the decompression process from the surface. As the depth and time of diving increases, more and more problems arise [2], including:

- the search for effective breathing mixtures,

- creating comfortable conditions for decompression for divers by choosing the appropriate technique and implementation of the course of diving,

- meeting the requirements of occupational safety and health of divers.

Determining the values of these parameters for a given diving system requires a preparatory process and time to acquire empirical data. Analysis of the processes taking place in the elements of the diving system during decompression and their simulation during the process of preparation, allows to indicate the factors determining the safety of diving.

Diving system is a complex anthropotechnical system in the system of interaction between the divertechnical security - maintenance - environment, the basic indicators and parameters of which are given in the technology or procedures developed before the commencement of underwater works. The combined and mutually dependent influence of technical parameters, diver characteristics and parameters of the water and hyperbaric environment on the course of the diving process, and in particular on the process of safe decompression, is the essence of the problem of the safety of underwater deep-sea works.

\section{AN IT REVOLUTION IT REVOLUTION IN DIVING CAUSED BY THE INTRODUCTION OF A PERSONAL DIVE COMPUTER}

As in all other fields of human activity, electronics and computerization entered the area of diving with the impetus about thirty years ago, and continue to impose constant and regular changes, along with the progress of their own active development [1]. From the very beginning, the process of using computer science in diving concerned in particular the decompression of the diver. This computerization was aimed at controlling and protecting the divers against irregularities during the decompression process. A dive computer is called a personal computer and in its application it is a tool provided to a given diver.

The dive computer market has developed, and continues to develop, thanks to one crucial advantage of these devices, which is the ability to adjust dive parameters in real time and to precisely plan dives (depending, of course, on the level of knowledge of algorithms by the dive computer user).

The diving revolution brought by the personal diving computer for air diving with the use of open-circuit breathing apparatuses was adopted and developed in the field of recreational diving. In a further stage of development, computers for diving with breathing mixtures and closed-circuit devices were introduced. With great formal resistance, the personal computer was accepted in other fields of diving, especially diving for commercial purposes. So far, it has still not been formally introduced in commercial saturated and deep dives [8]. In military use, personal computers have appeared in a specialized version for semi-closed and closed circuit devices, which, by the normal turn of events, resulted in their use also in technical recreational dives.

Currently, the personal computer is used on a mass scale in dives at medium depths, and in deep technical dives. Modern divers cannot imagine diving without a computer and data transfer about its course. The dive computer, in the form of a watch or console, replaces the functions of equipment that a diver would once have to use, such as a dive watch, depth gauge, cylinder manometer, reserve device and a plastic decompression table. In addition, the computer calculates and controls the decompression, records the dive course, measures the water temperature, alerts if the set values are exceeded, monitors the supply of respiratory gases depending on the selected option, etc. For the purposes of decompression assessment, it allows for the validation of its model, by assigning it to the personal characteristics of the diver. The dive computer provided the possibility to computerize decompression data. For the first time a wide range of divers take part in the decompression model research, and scientists have been provided with the opportunity to collect a "huge" amount of data and their statistical interpretation. Algorithms in dive personal computers were created not only on the basis of existing, modified or new models of decompression, but also on the basis of diving practice. The software for these computers is 
developed by the most eminent scientists-practitioners, working in international teams in leading research centers.

The market for personal dive computers imposed competition in terms of matching divers' preferences. The trend in the market of personal diving computers have become the standard in all areas of diving. The market operates on the basis of sublime marketing and intensive advertising aimed at persuading to buy the product of a given company and to praise the particular computer model. Computers compete with each other with the number of recorded parameters, multitude of models and the range of decompression algorithms, ergonomics of workmanship and ease of use, as well as the possibility of registering the course of diving. [8]. The excess of information contained in the dive computer is to "stun" and reassure the buyer of the computer for the price of several hundred or even several thousand dollars that it is the best and most fashionable equipment available on the market.

An average diver, a recipient of a computer, even interested in the subject of decompression, has considerable problems with assessing the full value of the computer. It is difficult to find any imperfections of a dive computer without knowing the roots of its construction. For example, what does the information about the programmes used for the decompression model of Bulmann's ZH-L12 or ZH-L16 algorithm, ZHL-8 ADT MB or the Buchanan ZHL-16C algorith with Gradient Factors say [9]. How to judge which algorithm is safer? The answer to this question is very difficult, that due to the requirement to possess considerable knowledge, with limited access to the results and methodology of research. It is all the more difficult because the authorised centres constantly correct decompression tables, thus improving the course of decompression models (usually a letter or a number is assigned to the model name). Every research centre that develops algorithms in a given model will promote its work. Centres and companies, on the other hand, fight to ensure that their expenditures on science and technology implementation yield economic success.

An important effect of the dive computer revolution is that it forces people working underwater to learn to master and use the capabilities of the computer and to search for knowledge about its operation, not only at the national level, in order to gain knowledge about safe diving.

Since its introduction, the personal dive computer has been the cause of conflicts and intellectual and organisational divergences in diving, which divided and still divide experts on the subject [10]. These are:

- indication of which decompression tables are safer - 'paper tables' issued in printed form or tables uploaded to a personal computer,

- indication which computer is the leading computer for diving - in a group of divers using computers with different models and decompression algorithms,

- indication, who better realizes decompression a person managing the dive from the surface, or a personal computer managing the dive decompression directly at the depth,

- indication which optimization of a given dive is more effective - performed by a supervisor or a personal computer.

Problems in commercial diving at the level of service companies and institutions are solved by the principle that it is the human being who decides and the computer is only to provide support.

Establishing this support is the most difficult problem to solve, depending on the organisation of underwater works, safety technology and procedures in accordance with the interpretation of the regulations in force. Choosing a personal computer for a given company or institution is connected with standardizing and targeting the implementation of underwater work, and with assuming legal responsibility. Polish legal environment does not regulate the use of a diver's personal computer for underwater works $[11,12]$. At present, there is only one possible compromise on the market of underwater services and activities in our country. The use of a dive computer is a personal decision of a diver, the taking of which has an undeniable advantage, because it allows for registration of dives, and constitutes its electronic Log Book [1].

\section{THE CHOICE OF DEEP DIVE DECOMPRESSION AGAINST THE BACKGROUND OF ADAPTIVE DIVE COMPUTER DECOMPRESSION ALGORITHMS}

In commercial deep-sea works in Poland, decompression is carried out on the basis of official "paper" decompression tables, imposed by the Act of 17 October 2003 on the performance of underwater works. On the basis of these tables, technical and material protection is planned and organized, and it is verified whether the diving technique to be used is suitable for the execution of given underwater works, and especially for decompression. In the decompression tables, the depth for selecting the diving decompression method is given in intervals of $3 \mathrm{~m}$ and the length of stay at the depth in intervals of 10 or $15 \mathrm{~min}$. Based on these intervals, the supervisor selects the actual depth and duration of the diver's stay. The structure of the tables allows him/her to select decompression only from the above intervals. For instance, the method and time of decompression are the same for a diver's 16 minute stay at a depth of $81 \mathrm{~m}$ and for a 29 minute stay at a depth of $83 \mathrm{~m}$ [2].

The method of selecting decompression using a dive computer is radically different from the one shown above. In this case, it is the computer that selects and supervises the decompression, and the diver may only enter the specific dive parameters, which are allowed by the algorithms of the computer.

In commercial diving and military diving, the computer has been introduced to a limited extent. The official reasons for this lie in the inertness of the institutions implementing regulations and supervising underwater works. The introduction of the computer would cause great legal difficulties, as it would imply choosing a personal computer for standardization, changing the organization of diving in the direction of lowering the role of the underwater works supervisor, and taking over the decompression by the diver proceeding according to the recommendations of the computer. What is more, the knowledge about decompression of an average diver would have to be radically extended in order to fully use the possibilities of the computer, especially in emergency situations, when he or she would be under severe stress.

The personal computer does not take into account diving technology, which is required by documents and professional diving regulations. Although 
the dive computer is not mentioned in the commercial diving documents, it is used as a duplicate of the personal LOG BOOK of the diver registering his/her dive. The supervisor of underwater works records the course of diving in paper form. As practice shows, it is often supported by data from the personal computer of the diver [13]. The dive computer is legally recognised as evidence in court, in cases of dive incidents, not only in recreational dives. Its usefulness is undeniable during the registration of dives, and in the worst case, it constitutes a significant evidence during the investigation of a diving accident [8].

A special class of diving computers consists of computers for deep diving. For the purposes of the article, for the definition of deep-sea diving I assumed "dives deeper than $45 \mathrm{~m}$, with the use of breathing mixtures" (a compromise between all regulations in the field of underwater activities). Contemporary computers for deep dives in an extreme case "cover" dives to a depth of $300 \mathrm{~m}$ [9], which in the opinion of the author and professional divers is madness in a race for risky record-breaking reaching of depths by "prominent" technical divers. As far as commercial diving is concerned, for depths in $300 \mathrm{~m}$ zones, it has no practical application. In these zones, saturated dives with technical protection are used, which can only be afforded by leading underwater service providers.

Depths greater than $200 \mathrm{~m}$ in technical dives are the depths that are reached by few divers, with outstanding psycho-physical condition and extensive experience. Their descent with the time of stay of a few minutes or only to the moment of reaching the depth, takes place with organizational, technical and medical protection significantly exceeding the typical protection of commercial underwater deep-sea works [4] (it does not apply to saturated dives).

Decompression of currently conducted technical dives (in our country according to the rules governing recreational dives), for planned and real depths and times of stay at depth, is calculated by the computer with an accuracy of $\pm 0.2-0.1 \mathrm{~m}$, with intervals of data downloading from 1 to 15 seconds. The diver's ascent, according to the calculated decompression is also controlled by the computer, for mixtures and oxygen prepared in cylinders (if its use is planned at the last decompression stop or stops).

To sum up, the algorithms and software of personal computers for deep dives, in addition to decompression calculations for various models and variants, have algorithms for the course of registration of extended data on the diver and the course of diving. Below I present the functions found in modern models of computers for deep dives [9]:

- diving planning,

- taking into account acquatic environment data,

- taking into account diver's physical built and level of training,

- the option to switch the working principle of the device from closed to open circuit and vice versa,

- selection of the model and decompression algorithms, for instance: ZHL-16C, VPM, DCAP, DCIEM, VVAL-18M, RGBM ) [9,4],

- optional calculation of decompression of breathing mixes for air and nitrox, trimix or optional heliox mixtures,
- assessment of the risk of a decompression incident, e.g. based on the level of micro-bubbles in the diver's blood, pulse measurement or body temperature readings. The computer calculates and selects the algorithm to adjust the so-called conservatism, which increases or accelerates the decompression time,

- $\quad$ assessment of the risk of oxygen poisoning by monitoring and calculating the so-called 'oxygen clock' in \% of the risk of the occurrence of central oxygen toxicity ('CNS'),

- recording of diving parameters such as depth, water temperature, oxygen partial pressure, optionally also carbon dioxide (semi-closed and closed circuit devices) and other, depending on the computer model,

- $\quad$ alerts of exceeding ascending and descending rates, diving operational times including decompression, and other,

- decompression recalculation in case of omitting a decompression stop,

- indicating directions of movement of a diver (compass) with roll offset,

- $\quad$ recording of parameters as a function of time up to several hundred dives,

- transfer of the above mentioned data to other IT devices for storage purposes.

The above list of functions shows that the software of modern personal computers, particularly those designed for deep diving, is already used at an expert and adaptive level, with indications developed for states of violation of decompression parameters. The programmes work in real time and introduce recalculations and decompression corrections for selected factors, especially important in commercial dives.

\section{SELECTION OF DECOMPRESSION BASED ON DIVING} PROFILE

The diver occasionally stays permanently at one depth, and the personal computer is able to easily take into account the changes occurring in the zone of the "planned target depth" of the stay. In the first models of personal computers, decompression schedules were entered from "paper tables", and the computer selected them, similarly as it is selected from these tables, i.e. for maximum diving depth. Only limited number of "paper tables" allow to take into account changes in the time of stay and depth of work of a diver in commercial dives. In this case, multilevel dives are introduced.

These dives have limitations in their use, and in practice are very rarely used. In the course of his many years of diving practice, the author has not seen any tables suitable for this type of diving in the case of deep-sea works. The resulting depth must be calculated before diving, as well as the choice of decompression method. Limitations of this method result from the fact that the depths of subsequent dives should be arranged according to the scheme from the smallest to the largest, and the last depth in the sequence should be deeper than the first stop of the assumed decompression (in our country the existing tables for multilevel diving are dedicated for air dives) [5].

For deep dives, the computer calculates and controls the decompression for a given profile of depth changes in real time. With this feature the decompression time can be significantly reduced, which is essential for the 
safety of a dive conducted entirely in the depths of the water, not only for technical dives, but also for all other types of dives. Long stay in the water depths threatens overcooling of the diver, which is an exceptionally unfavourable factor for the course of decompression. Low water temperature is a factor that necessitates the extension of the decompression process.

\section{MONITORING OF THE PHYSICAL LOAD OF A DIVER}

The physical effort of a diver is indicated by a computer that counts the number of heartbeats per minute, the consumption of the breathing mixture, the level of oxygen in the breathing circuit of the device, etc. The choice of decompression is strictly dependent on the diver's effort. The personal computer can take this into account by calculating a new decompression (provided the settings change), or by option switching to a decompression programme with an appropriate lower of conservatism, increasing the degree of saturation of the leading tissue in the decompression [9]. In turn, in case of hard work of a diver, with the use of "paper tables", the underwater manager himself switches the basic decompression to the so-called "prolonged decompression", i.e. the decompression from the next, longer period of stay at a given depth, or for a given period of stay, usually assumes the depth by $3 \mathrm{~m}$ greater.

The above functions performed by a personal computer would be very useful in commercial diving. Professional dive teams use more or less complex computer programmes to support the process of diving and decompression. These programmes, as a rule, solve one or more problems occurring during underwater work. In general, they can be assumed to be used in preparation for deep-sea underwater activities. The underwater works supervisor during the dive is mainly focused on the divers' performance of the task, the operation of the diving system operating personnel and the team securing the work from the surface. By obtaining data from a personal dive computer, the supervisor would be able to objectively evaluate the diver's performance and optimise the decompression process if, for example, the diver's planned working time is prolonged.

MONITORING OF DECOMPRESSION PROGRESS IN UNDERWATER DEEP WATER WORKS

The diving systems from which underwater deep-sea activities are carried out generally do not allow registration of diving parameters. National underwater regulations require recording of underwater diving conversations between divers and their supervisors. In 2005, while modernizing the domestic Af-2 system, the staff of the Department of Underwater Works Technology at the Naval Academy developed and installed desktop computers with software for recording diving parameters, as well as basic parameters of the atmosphere and respiratory gases and sound, images from cameras from the diver's helmet, installed in the diving bell and hyperbaric chamber.

This solution made it possible to recreate the decompression process, however, only in the part carried out in the diving bell and hyperbaric chamber. The recording is intended for saturated dives and is also used for underwater works. Despite the fact that in many cases the registration was incomplete (without the first decompression stops), it allowed to compare decompression according to heliox "paper tables" used in country with the decompression recorded by diving computers during deep-sea dives [13].

In order to analyse the full course of diving, and in particular decompression, it is necessary to use data from a personal dive computer at the stage of diving when the diver is in the water. When comparing the decompression times in each case, the results of the comparison were in favour of "paper tables". It was caused by many factors, and the author presents some of them in the subsequent part of this article.

\section{"GOOD DIVING PRACTICE" VERSUS PERSONAL DIVE COMPUTER FOR COMMERCIAL DEEP-SEA DIVES}

The good diving practice is a set of rules that are applicable during diving. They are the result of many years of experience, often related to the loss of health and sometimes even life of divers. We often forget that in these tragic incidents, improperly performed decompression had its significant share [14]. Especially in the early stages of the development of underwater medicine and physiology, as well as diving techniques, lasting until the 1970 s, the number of diving accidents due to decompression errors had a drastic impact on the formation of the "good diving practice". This article deals only with the principles of this practice which pertain to decompression [6].

For commercial dives, decompression is handled by the underwater work supervisor, who takes full responsibility for its course and possible complications during decompression. Today's underwater works supervisor plans deep-sea dives with prior recognition of the underwater task location. Such reconnaissance is carried out by means of technical means as well as identification of hydrological conditions, by means of underwater hydro-location or television installed on an ROV vehicle. This allows for a preliminary assessment of the working conditions, and introduces the diver to the area and place of the underwater task force. Such activities result from the so-called "good diving practice" and in the past century were referred to as "diving scouting".

The partner organization of recreational and technical diving requires a minimum of a pair of divers and a safety diver [15]. In a pair of divers one performs the function of a leading diver. From the point of view of the organization of commercial diving, in this case the supervisor in charge of the dive is under water. It is selfevident that the entire team of divers should have the same type of computer, or at least use the same decompression model. This is very important when using computers during deep dives. Decompression in this case can be done in a group or individually with individual settings for a given diver in his/her personal computer.

Contrary to expectations, the introduction of dive computers did not increase the safety of diving. The computer does not capture all factors determining the safety of diving, including important individual characteristics, such as the condition of the diver's health, and his or her training. Nor does the computer take into account all the diving conditions that should be introduced into it by the diver using it, and especially his/her individual, substantive preparation. This is one of the many reasons why to date the diving computer has not formally appeared in professional deep dives. 
Implementation of decompression in deep commercial dives must follow the official decompression tables, which differ in their structure and model from the tables "processed" and calculated in real time in the dive computer. Nowadays, professional divers have their own computers, and sometimes "smuggle" them into the dive, and then keep them in a vessel with water in the diving bell and in the decompression chamber (the dive computer works correctly only in water) [13].

One of the main differences of decompression calculated in computers, in relation to the "paper" tables is that the computer is set to relatively high partial pressures of the so-called bottom and decompression mixtures, to shorten the time the diver stays in the water depth. Personal computers assume that the entire decompression will take place in the depths, and high partial pressures of oxygen are unfavorable from the point of view of the effort of a diver working underwater. The use of lower oxygen partial pressure in paper tables implies a longer decompression time, but simultaneously reduces the risk of a decompression incident. Paper tables require that decompression be performed in the bell, and in its extreme case in the medium and final phase, in a hyperbaric chamber, with the use of oxygen from a depth of $12 \mathrm{~m}$. This contributes to a high level of comfort and optimal conditions for decompression. This aspect is important for the safety of the diver, especially when decompressing after strenuous work.

In technical dives, divers have the possibility to "improve" the decompression calculated by the personal computer, by changing its mode, adjusting its conservatism (reducing or increasing the gradient value of tissue saturation pressure). The supervisor of commercial underwater works does not have such a wide range of possibilities. He/she can improve decompression only within a narrow framework of "paper tables", by selecting maximum partial pressures of inert gas or oxygen, and by modifying decompression procedures in emergency situations.

When using decompression tables, it is 'good diving practice' for divers to base their decompression profile on a maximum depth that is slightly deeper than that which they actually dived to, and to make sure that during their ascent they closely follow the table they are using, being sure to remain close to, but no shallower than, the depth which is indicated in the tables. For example, for the real measured and greatest achieved depth of diver's work of $81 \mathrm{~m}$, which is specified in the table, we adopt for the decompression the next depth indicated in the table, i.e. $84 \mathrm{~m}$.

The simplest explanation for this is that it takes into account the accuracy of the depth measurement. The situation is similar with respect to the time of stay. If the table shows the duration of the stay of 45 minutes, then the diver's work ends a few minutes earlier, to maintain the reserve of a possible interruption in preparation for ascent. This happens despite the fact that in skilful diving this time can be very short.

In professional deep diving we encounter three possibilities of decompression implementation, which determines the comfort of the diver during its course. The best execution kind is decompression in the bell and in the chamber, during which the diver enters the bell in the working depth zone. In this case, decompression takes place in the most friendly conditions both for the diver and for the decompression itself. It provides thermal comfort, enables removal of equipment and staying in diving underwear, without any constraints from the diving [3].

A case similar to the above conditions is decompression partially in the water depth, with a diver entering the bell at a selected decompression stop. The most unfavourable condition is decompression of the diver in water, in a horizontal or sitting position. It involves almost all negative impacts, which the diver avoids in the "dry bell", especially in the decompression chamber. This is the position the diver takes when decompressing in a wet bell. However, a typical position, necessitated during decompression in technical diving, is close to horizontal. Moreover, this position is the most comfortable from the point of view of compression of equipment mounted on the diver's body. ${ }^{1}$

The entrance of professional divers to the bell can take place at the depth of the bell's stop, or during decompression at a selected station. This is dependent on the time reserve at the depth. A delay with the planned time of stay at the depth of only a minute results, in deep dives, in prolongation of decompression, e.g. at the depth of $82 \mathrm{~m}$, when the planned time of stay is 45 minutes, if we extend the time of ascent by 1 minute in the case of official Polish tables [5], decompression is increased by 2 hours 25 minutes, as it is necessary to apply the next time indicated in the table for the diver's stay at the depth of 60 minutes.

During the longest decompression period, the divers are in full comfort following the transfer under pressure operation (TUP) from the bell to the hyperbaric chamber. Decompression time relations in the water, diving bell and chamber depend on the time spent at the depth of the diver's work and, most importantly, on the "efficiency" and "effectiveness" of the diving technique to achieve the assumed decompression. Moreover, this relationship is also dependent on economic factors and material and technical security. Optimization of the decompression process concerns the usage of heliox mixtures, and is particularly important for the work of the open circuit diving equipment, which is characterized by high consumption of breathing mixes. This is particularly evident when optimising the shortest time spent in the bell and the long decompression time in the chamber, i.e. when divers breathe a working mixture in the bell and decompression mixture in the chamber (a chamber filled with decompression heliox mixture).

Decompression in the chamber requires many hours of breathing with oxygen from inhalers (BIBS), from stops $12 \mathrm{~m}$ and shallower (e.g. in the $95-120 \mathrm{~m}$ depth zone breathing from inhalers lasts intermittently 4.5 hours) [5]. "Good diving practice" forces the diving staff to discipline the divers to follow the recommendations during decompression in the chamber. The most important of these recommendations is to maintain the tightness of the oxygen mask so that the oxygen content does not increase in the chamber. Periodic changes of position, moderate body movement to improve blood circulation, drinking fluids, i.e. diving body hydration, and preventing sleep of tired divers during decompression are also mandatory [3].

The advantage of the "good diving practice" compared to technical and recreational diving is the assessment of the psychophysical condition of the diver by maintaining continuous communication and observation of the diver (duplex communication and at least one camera observing the working diver). This solution reduces to a minimum the risk of improper decompression, and allows to monitor its process, without the direct involvement of the diver. This is a fundamental difference in comparison with technical diving. At this 
point the author would like to quote the "opinion" concerning deep-sea technical divers, shared by the "veterans" of commercial deep dives. It boils down to one short, and yet very blunt sentence, "They are madmen". This assessment is not easy to reject, because the activities of deep-sea technical divers and their diving method are associated with high accident rates with fatal consequences [16]. In the case of emergency states and disturbances in the course of decompression, a professional diver interacts with the base on the surface, which provides him/her with better mental comfort, something that a technical diver is deprived of.

\section{SURFACE DECOMPRESSION2 OUTSIDE THE} CAPABILITIES OF A PERSONAL DIVE COMPUTER

In most of the cases covered by "paper" decompression tables, surface decompression applies to medium depth dives. Technical and organizational protection of technical and recreational dives offers limited possibilities for surface decompression. Nevertheless, this method of decompression, despite the criticism of some researchers, was introduced to deep dives in military applications, which are also used by commercial companies. In the last thirty years, tables of surface decompression for deep heliox dives have appeared in the US Navy and Canadian Armed Forces worldwide $[17,18]$.

Surface decompression for deep dives requires oxygen decompression in the water at the last stop of $9 \mathrm{~m}$, and the US Navy tables require a $12 \mathrm{~m}$ stop using a heliox mixture containing $50 \%$ oxygen. The same applies to Canadian tables $[17,18]$. To secure the surface decompression of deep dives under the conditions of deep underwater works, technical precautions are required to ensure oxygen decompression in the water at the stops from $9 \mathrm{~m}$ and below or to use a wet diving bell with the possibility of performing such decompression and a decompression chamber for the continuation of oxygen decompression.

In an emergency situation requiring a rapid ascent of a diver to the surface, e.g. in cases of flooding or a major leakage of a diver's suit, we are compelled to decompress at the surface. Such cases have occurred during deep underwater works carried out with the participation of the author, in structures with sharp protruding elements. Even when applying detailed working checks of divers' equipment, utilising the best and tear-resistant diving suits, such a situation cannot be ruled out. In these two situations, the dive was carried out with an open (dry) bell, and the diver, after damaging the suit, assisted by a colleague, found himself in the "dry" atmosphere of the bell within 2-3 minutes. Therefore, the author is of the opinion that a pair of divers in the water depths during deep-sea diving is, in accordance with "good diving practice", the best organisational option.

\section{POSSIBILITY TO SUPPLEMENT DATA FROM A PERSONAL DIVER'S COMPUTER FOR PROFESSIONAL DEEP_SEA DIVES"}

Professional deep-sea diving has not succumbed to the revolution in dive computers, and continues to be carried out in accordance with the level of knowledge and tradition of the $80 \mathrm{~s}$ of the last century. The currently used decompression in professional deep-sea dives in the world, originates from the same period of time, with some later modifications in the 21 st century, related to the implementation of decompression [2]. The use of only a part of the dive computer's capabilities to record the basic parameters in these dives directly from the diver, taking into account the depth, time and temperature of the environment, allows for the evaluation of both decompression and the entire diving cycle.

These data are of fundamental importance for the assessment of the performance of a diver and an underwater work supervisor, as well as for the work of technical support staff. The ability to plan and simulate decompression of the dive, taking into account the data of the aquatic environment, and the characteristics of the physical build and training of the diver i s also important. They will allow a more precise estimation of the needs of breathing mixtures and their supplies. The assessment of the risk of oxygen poisoning with the planned duration of stay at the depth of work will allow the selection of appropriate levels of oxygen content in breathing mixes [19].

In emergency situations, when a decompression incident occurs, ascent or descent rate is exceeded, a decompression stop is omitted or the duration time of decompression stops is violated, and when a decision must be made instantly, a diving computer is indispensable. The condition for utilisation of this signal is ensuring the communication of the above mentioned data to the underwater works supervisor, who controls the decompression process, and to the service personnel. At the current level of development of personal dive computers, such information may only be provided by the diver with a telephone, although the transmission of the signal from the computer over the telephone cable or TV of the harness of the diver's helmet should not be a technical problem.

Most dive computer options, especially adaptive ones, cannot be used for formal, legal and technical reasons. For example, the choice of decompression models and algorithms is not an option because the tables in force are assigned a model and decompression algorithms [3]. A deviation from the tables has legal consequences [11]. In professional deep-sea dives, open-circuit diving equipment is formally required, which does not allow to use the full range of possibilities of diving computers, which are designed for closed or semi-closed circuit devices.

Is it possible that a specialized dive computer for professional deep-sea dives will become available? The author estimates that currently, in underwater services, the demand for this type of computer is very small, and the formal obstacles are difficult to circumvent. According to the author, the first step will be to develop a computer to record the course of diving, operating both in the water and in the hyperbaric gas environment of the diving bell and decompression chamber. Such a solution would be beneficial for the diver's career, not only in deep-sea dives, but in all types of professional dives.

The prerequisite for such a solution is an amendment to the Regulation of the Minister of Infrastructure of 19 May 2004 on safety and hygiene at work while performing underwater works [12] and the Act of 17 October 2003 on the performance of underwater works, 


\title{
REFERENCES
}

1. S.Skrzyński „Diving computers - a revolution in diving organization” conference materials of the 2nd Scientific Conference PTMiTH2002S;

2. S. Skrzyński - "Historical outline of deep dives in Poland. Part I General characteristics of deep dives, Polish Hyperbaric Research, rok 2006;

3. S. Skrzyński and team Target project No. 11 / BO Contract No. 148308 / C-T00 / 2001: "Deep-sea diving for the needs of the Sea Rescue". Analysis of foreign deep diving systems in the aspect of domestic conditions;

4. Exchange of views on deep dives with foreign and domestic specialists;

5. Regulation of the Minister of Health of 17 September 2007 on health conditions for the performance of underwater activities;

6. NOAA Diving Manual" Diving for Science and Technology US Department of Commerse USA 2002;

7. Sygn. Mar. Woj. 860/81: Divers decompression and recompression tables. Navy Command,Gdynia 1982;

8. S.Skrzynski, A. Olejnik, S. Poleszak „Analysis of the use of Dive Computers by Divers MW RP conference materials of medical and technical problems of diving 1998;

9. Comparison of deep dive computer data produced after 2016 companies:Scubapro, Shearwater, Suunto, Scubapro, Oceanic, Mares, SEAC;

10. Michael Menduno "Anatomy of a Commercial Mixed-Gas Dive” "Alert Diver Q3 Summer 2018;

11. Act of 17 October 2003 on the performance of underwater works;

12. Regulation of the Minister for Infrastructure of 19 May 2004 on health and safety at work in the performance of underwater works;

13. Own data from underwater deep work on the Polish shelf and developed procedures and executive documents regarding the implementation of deep dives;

14. The professional diver's hand book John Bevan SUBMEX 2005;

15. T. Mount, B. Gikiam., ,Mixed gas diving" Watersport Publishing Inc. San Diego USA 1993 ISBNO - 922769-41-9;

16. Richard L. Pyle, Ph "Toward A New Era In Recreational and Technical Rebreather Diving " Rebreather Forum 3 Proceedings 18 - 20 May 2012 ;

17. US Navy Diving Manual". Published by Direction of Comander of Navy. Revision 7, 2016;

18. DCIEM Diving Manual part 2 Department of National Defence 1995;

19. S.Skrzynski „Modern principles of divers decompression modeling in the aspect of their application” AMW 1998.

\author{
Stanisław Skrzyński \\ Akademia Marynarki Wojennej \\ im. Bohaterów Westerplatte $81-103$ Gdynia 3 \\ ul. Śmidowicza 69 \\ tel.: +58626 2746 , \\ e-mail: skrzynski@interecho.com
}

\begin{abstract}
${ }^{1}$ At this point, we should recall a rule, forgotten and not obvious to many, concerning the point of a diver's body, on the basis of which we determine the depth of his/her descent. This point is the point of buoyancy (centroidal) of the lungs, representing the pressure in the lungs, and according to which the depth for decompression calculations is determined. In most cases, in underwater work, divers remain in a slanted position, close to an upright position, also during decompression (the specificity of helmet diving). In this position, the difference in pressure between feet and lung buoyancy point in practice is about 1.2 water column, which corresponds to $120-160 \mathrm{kPa}$ or $0.120 .16 \mathrm{bar}$ ). This results in compression of the diver's body, and increases heart rate to ensure blood circulation. The inclined position, so natural for a working diver, reduces this difference. However, in a close horizontal position, the difference is in the range of 0.1 to $0.15 \mathrm{~m}$ water column. The nearly horizontal position of the diver in the water depth is optimal, both for cardiovascular and lung function, due to the lowest respiratory resistance. Correct functioning of the diver's circulatory and respiratory system is essential for safe decompression.
\end{abstract}

2 Surface decompression is a method of decompression, in which the diver performs part of the decompression in the depth of the water, and at a specified decompression stop or at shallower stops, the diver performs decompression on the surface, in the convenient conditions of the decompression chamber. The time of transition from the stop in the depth of the water to the chamber, is strictly regulated, and ranges from 4 to 5 min. The faster the transition occurs (without excessive haste and effort), the lower the risk of a possible decompression incident. Surface decompression can be realized only up to selected depths and times of stay. It is forbidden in case of intensive work and difficult diving conditions, including dives at depths of e.g. $300 \mathrm{~m}$ below sea level. Depending on the decompression tables, surface decompression constitutes the standard decompression method in use. The benefits of said method of decompression, with the exception of forced circumstances, are experienced by divers in two cases. The first case is when the scope of work is very large and there is a need for appropriately fast replacement of the diver at the workplace. Conducting several different decompressions in water is very difficult and risky for the diving supervisor. The second is the case of training dives, when surface decompression allows to "let" a large number of divers to the adaptive depth of the body to the pressure. The exception are tables from the Regulation of the Minister of Health of 17 September 2007 on health conditions for the performance of underwater works, which reads that "Surface decompression is used when decompression in water creates a risk of loss of health or life of a diver (currents, temperature, explosions)". The procedure of surface decompression was widely used in our country until the 1990s. The introduction of the Act on underwater works for air or nitrox diving in operational dives at medium depths resulted in the abandonment of the use of surface decompression. 